\title{
Fragmenting Techniques through Form Finding Process Towards New Architectural Form
}

\author{
Eng. Sarah Ismail Hassan Soliman \\ Teaching Assistant. at Arch. Department \\ Faculty of Engineering-Mataria
}

Prof. Dr. Mohamed Alaa Mandour

Professor of Architecture at Arch. Department

Faculty of Engineering-Mataria

\author{
Asis. Prof. Hala Adeeb \\ Assistant Prof. at Arch Department \\ Faculty of Engineering-Mataria
}

\begin{abstract}
:
It can be considered that there is rareness equivalent reaction in the academic field on the theoretical analysis for the new techniques in designing complex and morphed architectural forms. Therefore, the research aims to clarify out the most important techniques that depended on fragmentation in the architectural form to investigate the underlying cause for the new distinctiveness forms of the recent architecture era that depended on the chaos and fragmenting concepts. A content analysis is conducted on the new forms, which aims as well as to find out how these techniques can be applied in regard to the form conception to give it a new transformation features on the architectural forms. In this context the research will determine the main fragmenting techniques that cause the form to be break, divided or fracture, through illustrating each technique, and how it can be applied, and determine the most techniques that fragment and transform the whether the whole form or affect and transform only the form external skin.
\end{abstract}

\section{Introduction:}

Linguistically, the word fragment is drawn from the Latin fragmere, whose primary meaning is 'to break.' A fragment is thus a piece or, strictly, a piece of debris. Accordingly, it can be considered that fragment is necessarily incomplete and subordinate to the whole that completes it. Moreover, fragment is considered to be as jigsaw puzzle, in which every side of the piece contoured so it fits into a general structural program. But it is clear that, while philosophical and architectural fragments do often lend themselves to such subordination, they are just as often able to resist the expected unity, their outer contours suggesting alternative puzzles. So that morphing process from simple form to a fragmenting one diverse according to the level of transformation, which determine the complexity value of the final fragmenting form. 


\section{Fracturing:}

It can be considered that nature always combines a great deal of irregular complex shapes which are oscillating and occurring changes, like what found in leaves, human body particles, clouds, ... etc. Thence the evolution of the mathematics and science achieving the fractal geometry, and the fractal cascades, which facilitate viewing strange and unique scenery and giant forms in nature as shown in fig- 1 .

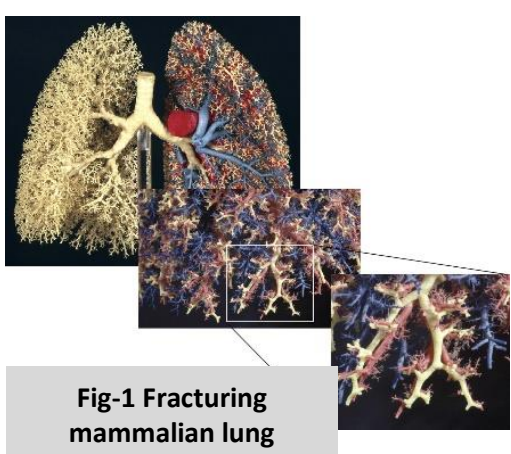

Fractals, this irregular forms that shows similarity in each level of magnification, can considered as one of the most effective technique in the architectural composition, whereas it gives a new feature to the architectural form like dynamic, unity and diversity, whereas, Fractals are self-similar sets whose patterns are composed of smaller-scales copied of themselves, possessing selfsimilarity across scales. This means that they repeat the patterns to an infinitely small scale. Fractal technique provides a quantifiable measure of the mixture of order and surprise in a rhythmic composition. Fractal geometry is a rare example of a technology that can reach into the core of design composition.

Nowadays fractals received many changes in its aims, characteristics, and the methodologies to execute it, which occurred based on the new architectural theories and techniques that developed during the new digital breakthrough and its givens in the various fields specially the architectural field, (Carl Bovill-2015).

\subsection{Technique Concept:}

Fractal forms are characterized as rough or fragmented geometric shapes that can be split into parts, each of which is (at least approximately) a reduced-size copy of the whole form, (Xiaoshu Lu-2012).

In this context, morphing process in fractal system can be illustrated through two main techniques as the following:

\subsubsection{Fractal Visual self- similarity:}

On any scale 'Since all levels of magnification, fractals are similar', as shown in fig-2 the self-similarity feature is a prominent characteristic of the fractal forms, whereas it indicates the similarity between the parts that form the shape with the whole shape, i.e. the part of the whole is so similar to that whole, (Altuhafi Assda-2015).

\subsubsection{Fractal Dimension:}

The regular geometrical shapes have dimensions as integers, while the fractal shapes have dimensions as a fractal number which expresses the complexity degree of the geometrical shape. Dimension is a scalar quantity that when the scale becomes smaller, represents how fractal fills the space. A pattern with higher fractal dimension is more

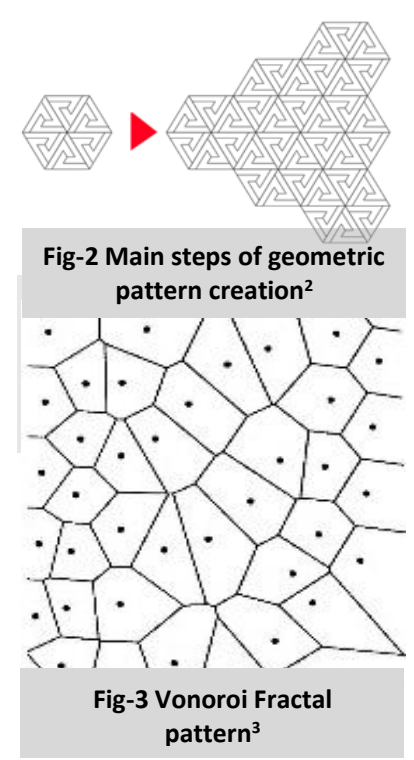


complex and irregular than lower fractal dimension and will take up more space, (Benjamin Aranda-2006). One of the most important examples of fractal dimension is vonoroi pattern as shown in fig-3, 4 . Voronoi diagram are of a class of fracturing called Dirichlet tessellations, irregular tessellation of the plane that occur spontaneously in nature at every scale and are used in the study of many fields including biology, anthropology and growth of crystalline structures.

One of the most important application for fractal dimensions can be observed in King Abdullah Petroleum research center project by Zaha Hadid, fig-5, that shows the complexity and fragmentation in the building form which consists of a unique cascade of fractals.

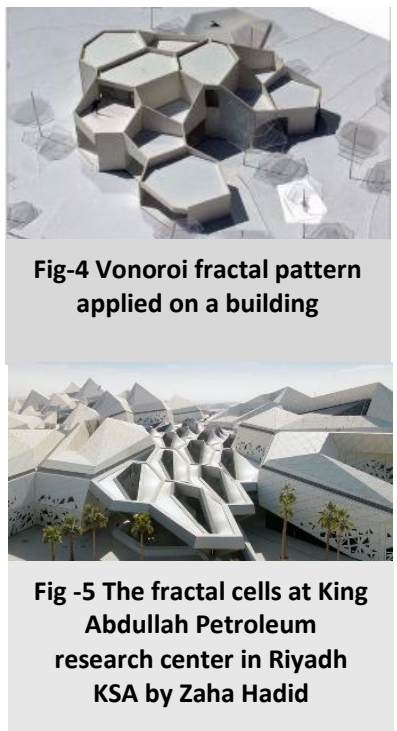

So in fractal preferences, the fractal dimension is more important than scale, because in any sizes, the fractal dimension is maintained and reflects the essence of fractals. This matter has caused fractals' application in science, (Walter" W. Piegorsch-2002)

\subsubsection{Fractals self-affine:}

Affine transformation is more general than a similarity, and dimension, whereas the affine shape can be deformed into irregular and random deformations, without disrupting the overall character of fractal system, for example affine
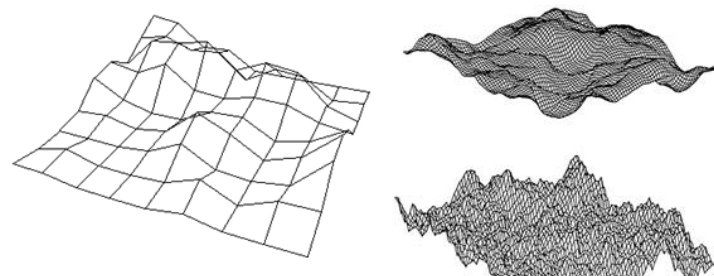

Fig-6 Three levels of deforming a self-affine fractal surface $^{5}$ transformations transform square into rectangles or parallelograms, and circles into ellipses. Thence a self-affine fractal form is one that is made up of smaller affine copies of itself which gives the self-affine system a greater ability to change and grow in nonlinear motions. Fig-6 shows a fractal surface based on self-affine system and how it gives the surface a great deal of flexibility and roughness, (Kenneth Falconer-2013).

\section{Erosion - Porosity:}

Erosion is broadly defined as a group of processes of eroding or being eroded by flowing agents, whether wind, water, or ice, which sometimes behaves like a fluid in the large mass of a glacier. Gravity pull may also influence erosion. So, erosion in nature can be considered as a continuous morphing process in macro and micro scale in form and pattern generation through deposition or subtraction of material, such as morphed stones by natural factors like wind, rain, etc. Thus, erosion, as a concept in the earth sciences, overlaps with mass wasting or mass movement through the transfer of earth material down slopes as a result of gravitational force, as shown in fig-7.

Nowadays, many Architects use these natural morphing processes as an inspiration to generate complex free forms and transition features that have never 
seen before, depending on the digital architectural breakthrough which facilitate unlimited tools and techniques in design and visualization process, in which it gives the ability to reach this level of

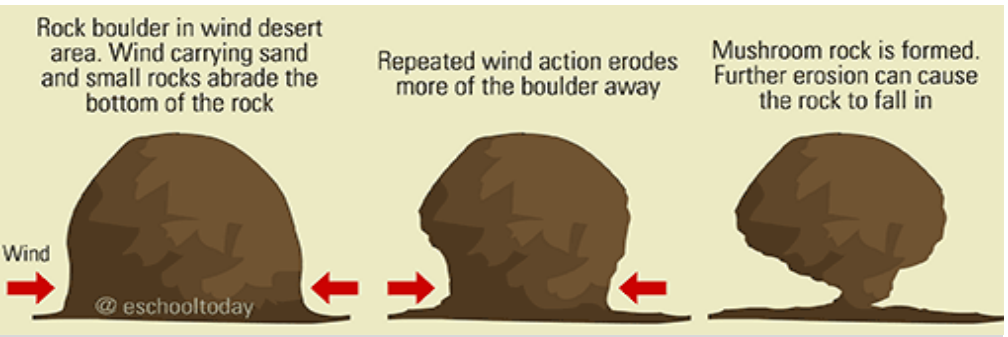

Fig-7 Morphing occurs in a rock through wind erosion process morphing and complexity that is found in erosion techniques.

In this context, there are many types of erosion that can be found in nature, which depend on the eroding forces and the affected material, such as; Wind, stream, rain, and costal erosion, which have been inspired in architecture through different techniques. So the research will illustrate the main concepts of these techniques, and how it can be applied in architectural forms, (Robert Stuart-2012).

\subsection{Technique Concept:}

\subsubsection{Wind Erosion:}

Wind collect materials as dust, that can be moved to be deposited, constantly eroding and reshaping rocks or sand dunes according to various wind conditions, which cause continuous transformations in its forms. This concept is applied in the architecture form through morphing and eroding in its outlines horizontal linearity fig-8, or through giving transformation by adding wide porosity to the form, the two techniques are shown in fig-9

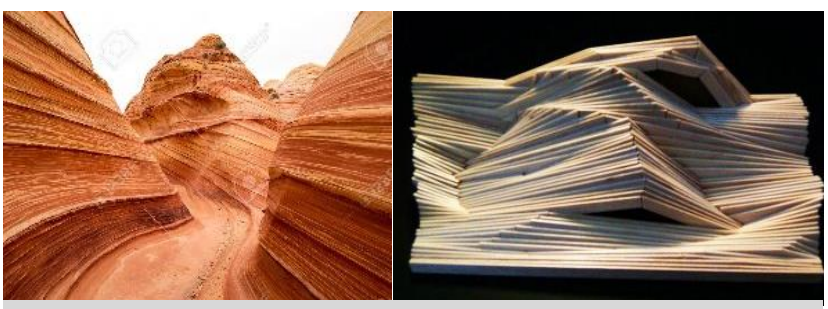

Fig-8 Linear Wind erosion in nature and its applications in architecture

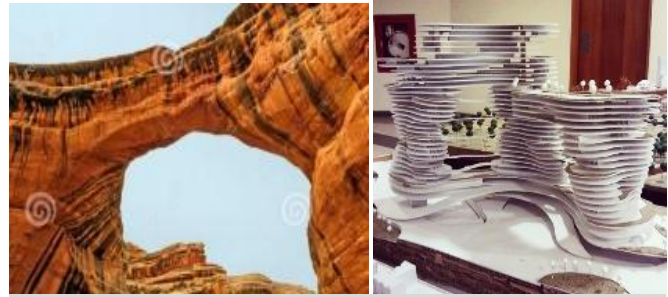

Fig-9 Wide porosity wind erosion in nature and its applications in architecture

\subsubsection{Stream Erosion:}

In rivers or streams, the faster the water flows, the greater the erosion effect. It takes place through two techniques: by water eroding the surface, and through the force of sediments eroding the surface. This concept is applied in architecture through form cracking erosion which divide the form through non-linear lines, as shown in Fig-10.

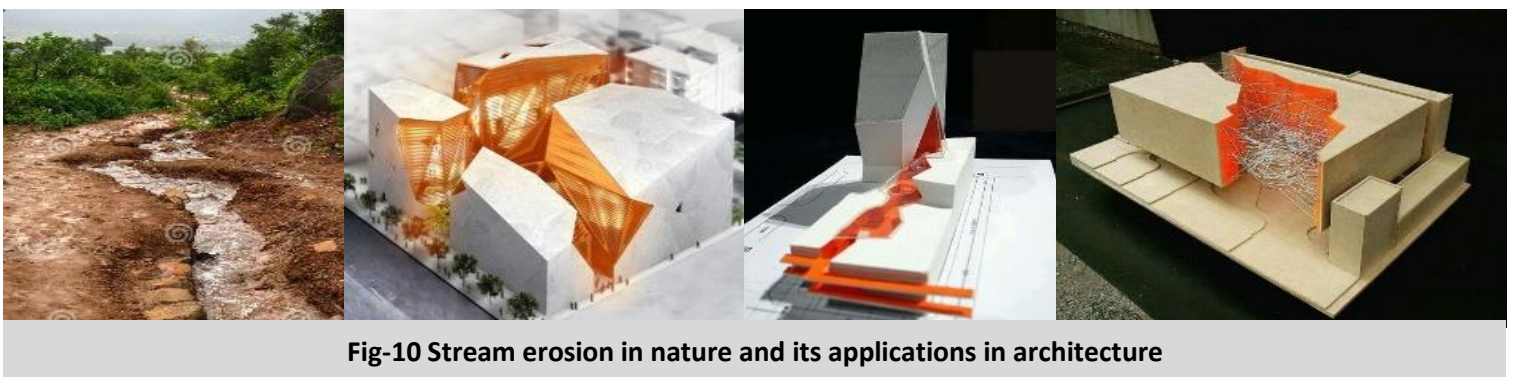




\subsubsection{Rain Erosion:}

Rain erosion has two stages, when these loose particles are moved with the flowing water, and when the impact of rain erodes the soil particles. This is applied in architecture form through vertical eroding and morphing in the form, or by applying small unregularly porous to the form outer skin. As shown in fig-11.
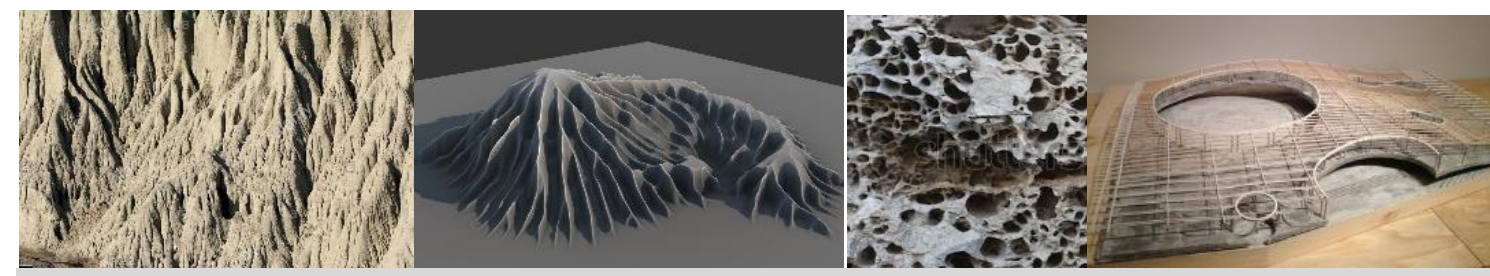

Fig-11rain erosions type in nature and its applications in architecture forms ${ }^{6}$

\subsubsection{Costal Erosion:}

This erosion is produced by the continuous strike of waves against the shore. Waves can erode rock or sand and cause wide porosity to their masses, this is applied in architecture form through creating wide void fractions "porosity" which morph the cubic forms from solid to porous as shown in fig- 12 .

\section{Triangulation:}

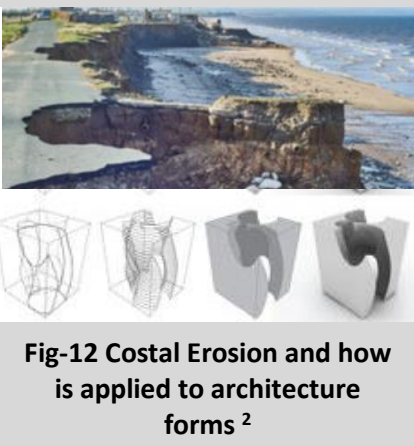

Triangulation is a kind of fragmenting the form through the division of its surfaces or a plane polygon into a set of triangles, usually with the restriction that each triangle side is entirely shared by two adjacent triangles as shown in fig-13. A surface with infinite number of triangulation is called compact surface.

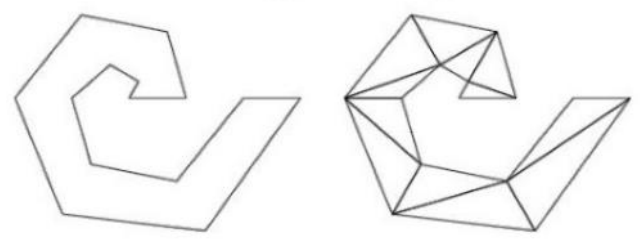

Fig-13 Trangulation ${ }^{2}$

The emerging use of Triangulation in Art, Architecture and Design was not just for visual impact, but increasingly for functional innovation. Triangulation is an ultimate solution, it is a very simple and stable shape, and it has been used in building structures for centuries. As in Eiffel Tower, its structure is a mesh of triangles.

The Systems of triangulation where multiple triangles are reiterated to form a network of structure and support. While triangulation systems have typically been utilized for their inherent structural properties, the prismatic patterns they engender also result in a matrix with great visual interest in building 3D Form.

Due to a digital paradigm in 3D modelling, the triangulation concept became more complicated, which is not only all done using mesh surfaces that are tessellated with triangles, but also the major digital architects have all recently been using striking triangulation features in their work. Architects have taken this aspect to another level, viewing this structural system simultaneously as a sculptural element and using it to envelop façades with striking aesthetic qualities. Whether triangles are used in even conformations to create a shell-patterned skin of a building, or they are 
employed in a variety of sizes to create 3D surfaces, spiky triangulation and triangulated patterned facades.

\subsection{Technique concept:}

There are many number of possible techniques that can be used to triangulate surfaces, here the research will illustrate the most common and important methods:

\subsubsection{Delaunay Triangulation:}

This method is based on the Voronoi Graph, and is created through connecting all generating points which share a common tile edge. Fig-14 illustrates this.

- Tessellation split a plane into a number of polygonal regions called tiles. (Vonoroi diagram).

- Each tile has one sample point in its interior called a generating point, all the other points inside the polygonal tile are closer to the generating point than any other.

- Delaunay triangles (red lines) and associated with vonoroi tessellation (black lines) for nine generating points are shown.

- Triangle edges are perpendicular bisectors of the tile edges.

- Points within a tile are closer to the tile's generating point than to any other generating point.

\subsubsection{Triangulating Cracking:}

Cracking generates a geometry of self-similarity of triangles trough any shape, in which the shape being recurred to another set of triangles. Recurring is about to return to origins within each new crack. Fig-15.

- Choose a shape to be cracked, and find its centroid.

- Create subsidiary shapes by connecting the centroid to each end of one edge of the parent shape.

- Repeat the previous steps for each new shape.

- Continue until a limit is reached, (Benjamin Aranda2006).

\subsubsection{Triangulating Spiky:}

Through polygon triangulating as mentioned before in this manner may have some "spikiness," such triangles may tend to be smaller and more localized and other can have kind if surface tension to give more "spiky" triangles in a triangulation produced by the conventional technique. As shown in fig-16. Thus, fewer pixels may be affected than in the conventional triangulation, and performance may accordingly be improved, (Donald P. Brown-2012).

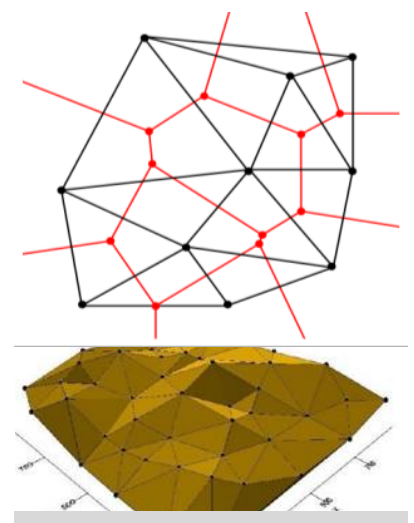

Fig-14 Connecting the centers of the circumcircles produces the Voronoi diagram

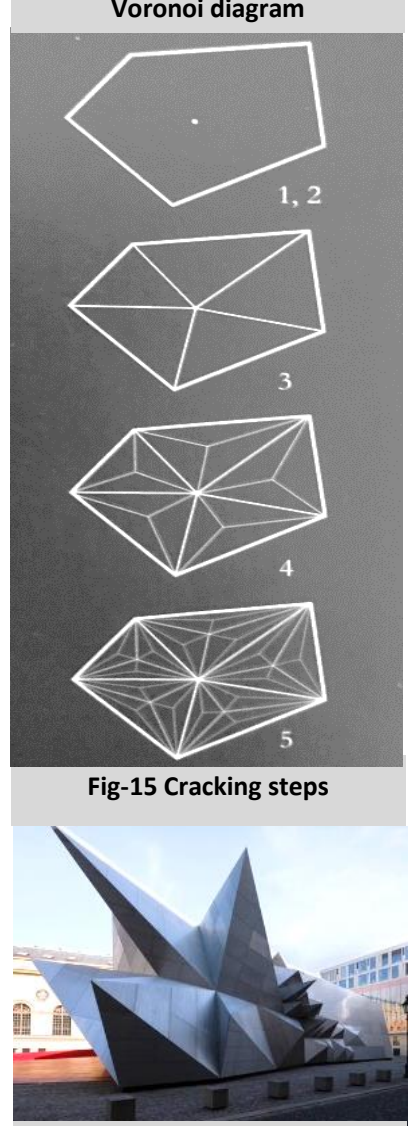

Fig-16Triangulation Spiky Wolf Prix - Pavilion Opera Space by Coop Himmelb(I)au 


\section{Composition/Assemblage/Fusion:}

Fragmentation through these techniques is a part of the deconstruction movement. The idea of these techniques is to take different shapes and volumes and morphing in the form through putting them together in unconventional ways so that they collide to form different shapes that in turn make the spaces of the buildings as in

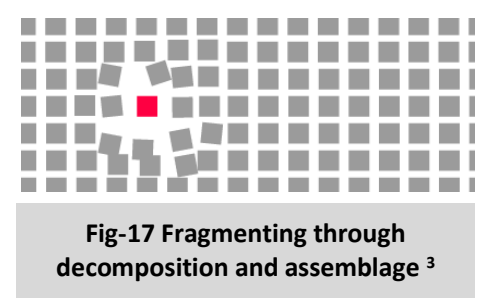
fig-17. Many architects used this technique referred to as "assemblage", which gave their buildings a design language of angles and tilted volumes introduced visual tension and ambiguities. The idea behind using the forms was to let the forms be the thing and to replace decoration, to get passion and feeling into the building.

This type of form finding architecture works together with sculpture. In which forms are a fusion of sculpture. Through this type of fragmentation architects used skewed geometry and creates heterogeneous, non-rectilinear, and curvilinear forms that are deflected, deviated, distorted, dislocated, and continuously deformed. Whereas these forms have no purity, repetition, or a sense of stability, but became contaminated by some sort of 'alien'. They have a different sensibility, as these buildings seemed to move, constantly change and to be instable, (Kevin Li-2015). This controlled chaos is a way of deconstructing harmony in architecture. Here the research will illustrate some of these important technique:

\subsection{Technique concept:}

\subsubsection{De-composition and Assemblage:}

This technique adopted structuralism as the starting point of composing and decomposing the forms in a way that impossible to understand the piece of architecture in the individual parts, but only in a context of unit. Each piece of architecture composition has a different function and has properties to be deformed inside a system and never individually, (Bruna Ferreira-2014). In addition to defining heuristics of morphing are fully reflected in the following:

- Negative heuristics: avoid rigid geometric primitives like squares, triangles and circles, avoid simple repetition of elements, avoid juxtaposition of unrelated elements or systems, as shown in fig- 18 .

- Positive heuristics: consider all forms to be morphed malleable, differentiate gradually (at

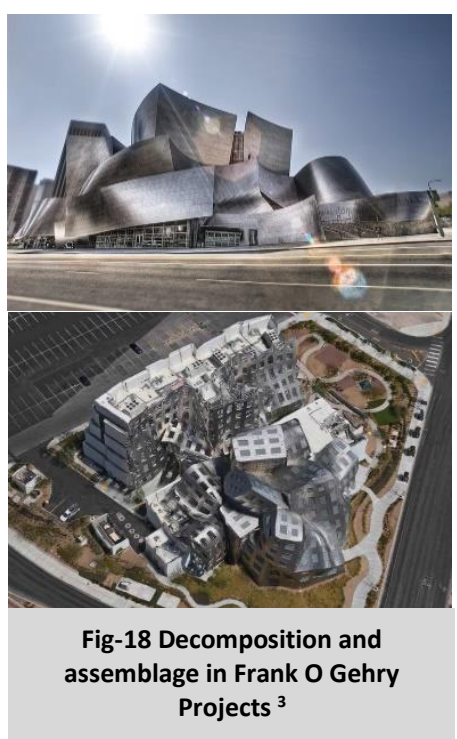
variant rates), inflect and correlate systematically. 


\subsubsection{Fusion:}

Fragmentation in this technique can be achieved through morphing that can be occurred on the axis, in which there is no single axis or hierarchy of forms, but a nest competing and confliction axes and forms, whereas the form geometry became increasingly irregular, (Philip Johnson \& Mark Wigley-2005), as in fig-19.

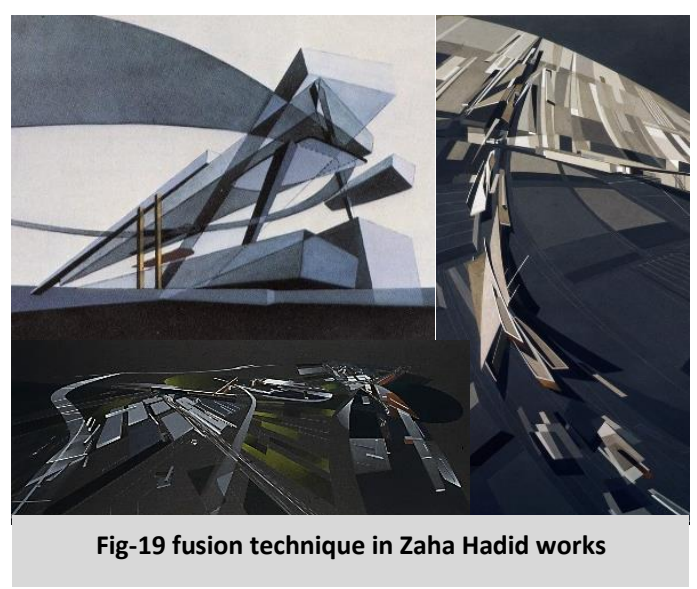

\section{Case Study: Analyzing Fragmenting techniques through a comparative study between Guggenheim, National Art Museum, Lo Monaco House:}

In this part the research will analyze these three contemporary projects from the global architecture that dealt with fragmentation concepts in various ways, aiming to illustrate and determine the most techniques that fragment and affect whether the whole form, or only affect the external skin of the form, which can help in putting a methodology for applying these techniques:

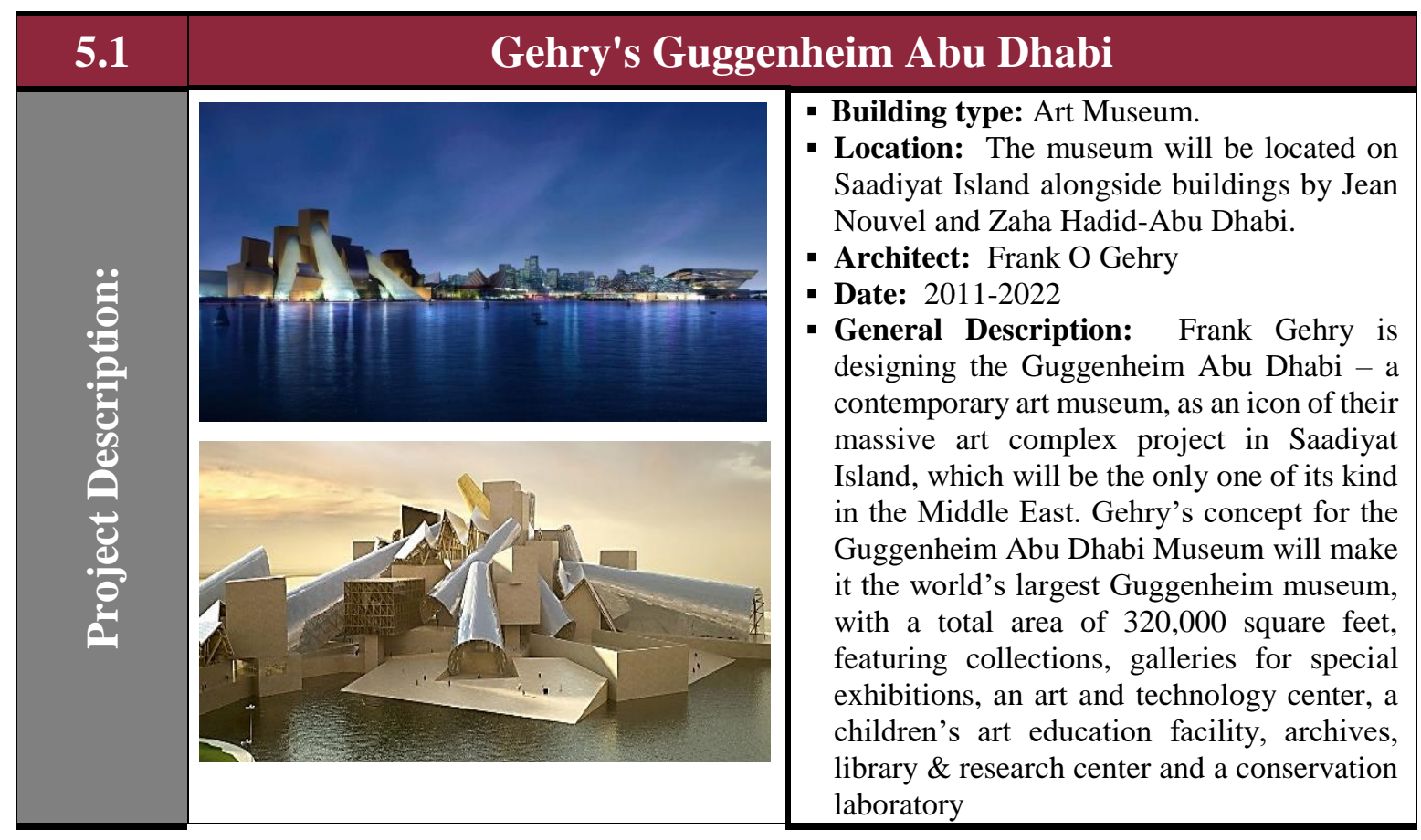



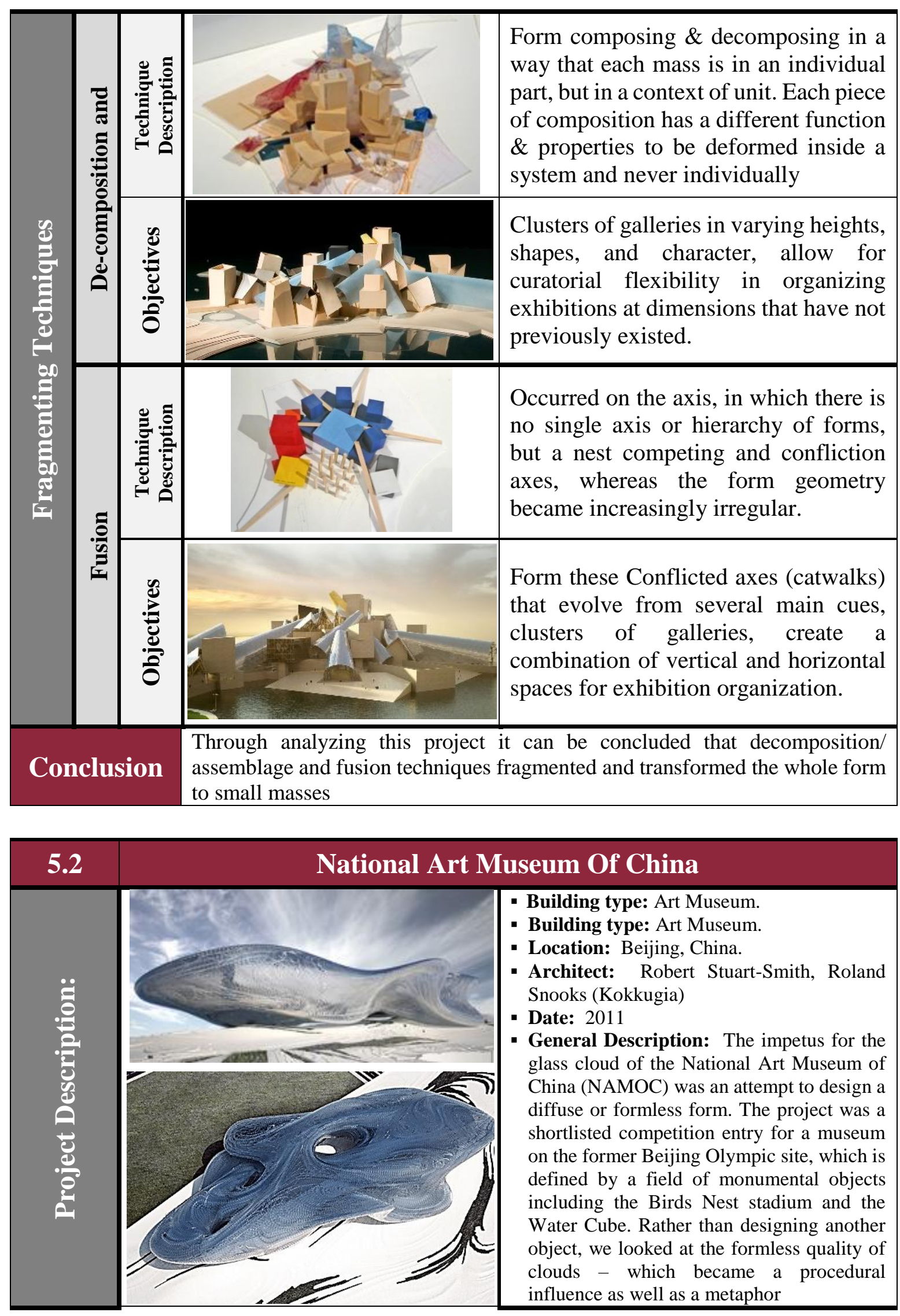

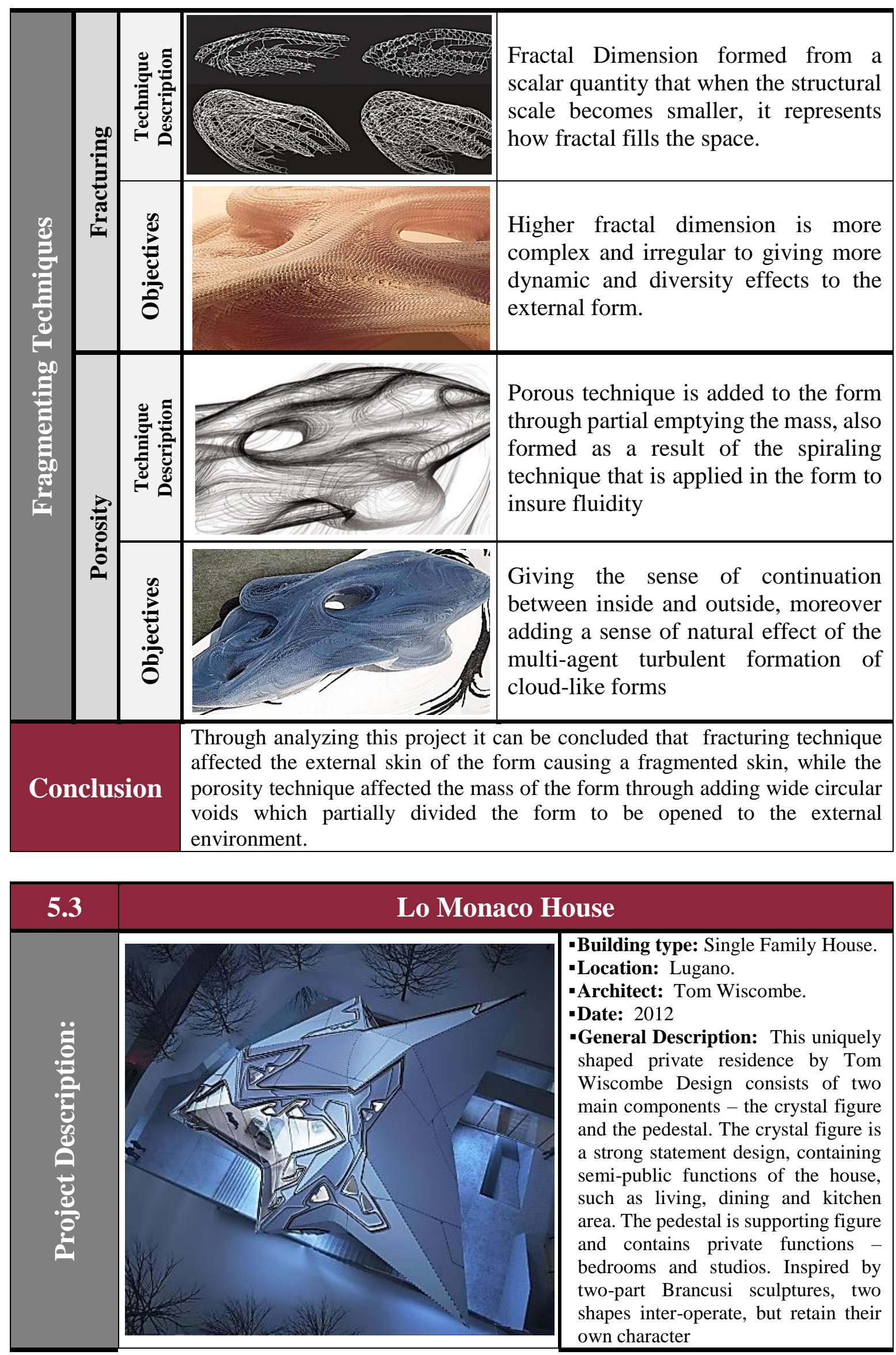


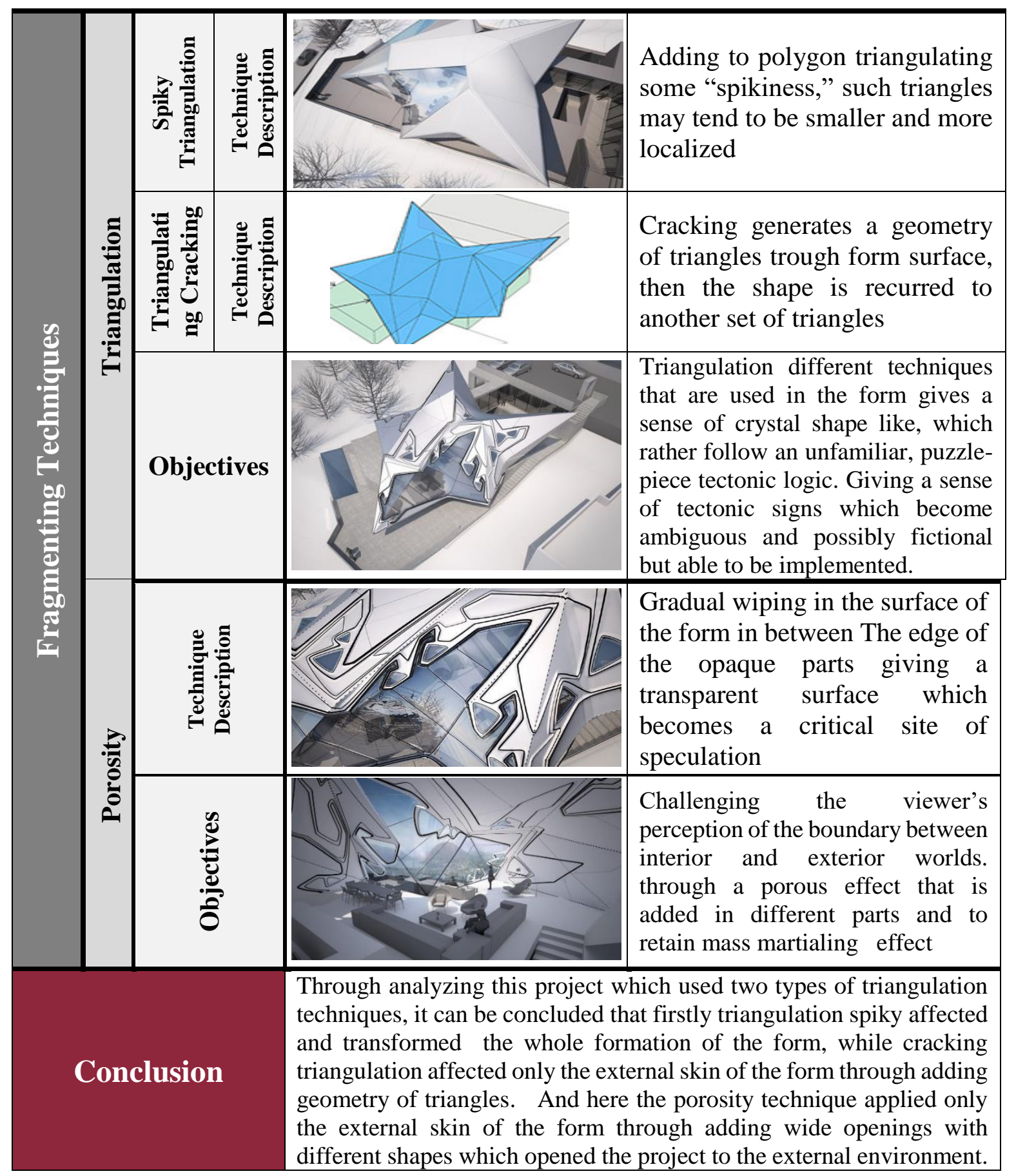

From the previous study to these three samples, the research can conclude different conclusions concerning how these techniques can affect and transform the form as the following:

- Fracturing technique transforms the form external skin.

- Porosity technique transforms whether the whole form, or form external skin.

- Triangulation techniques transform the formation of the whole form like triangulating spiky, and can transform only the external skin like triangulating cracking

- Decomposition, assemblage and fusion techniques transform the whole formation of the form to small particles. 


\section{Conclusion:}

The research tried to clarify the concept of fragmentation in the architectural form to reach the most important techniques that have been used recently in the architectural forms, that leads finally to reach a form that can be divided, broken or fractured through different ways.

Through the research and particularly the case study the research concluded that fragmenting techniques can be classified into two main classifications, according to how they affect the form, which will be illustrated in the following diagram:

\section{Fragmenting Techniques Classifications}

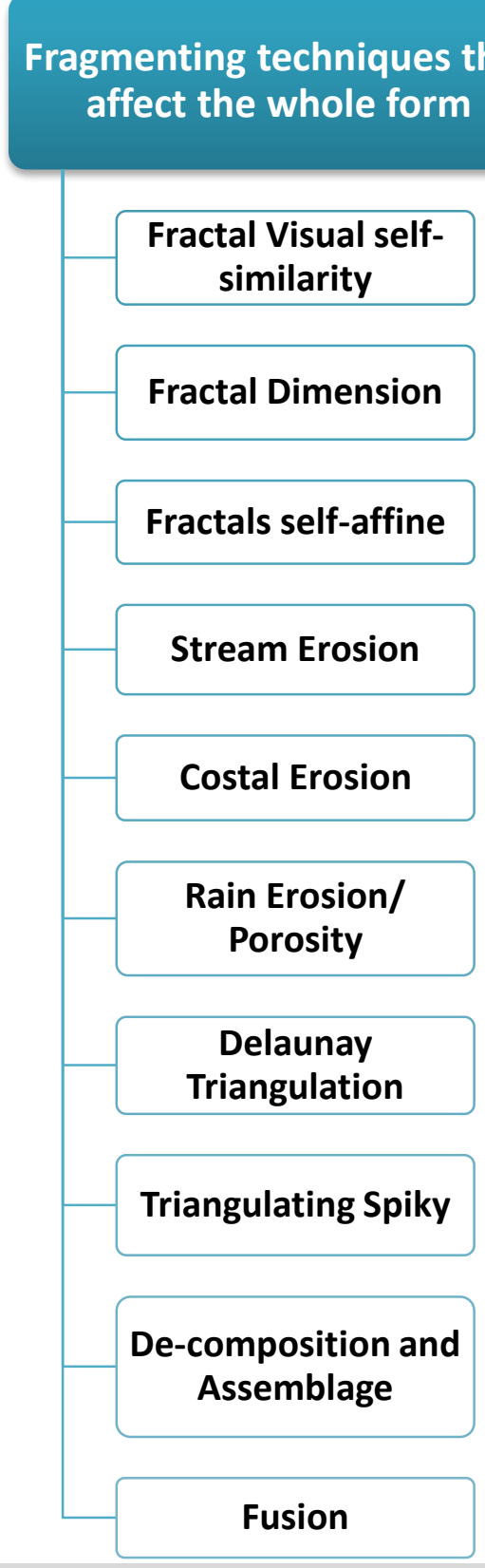

Fig-20 fragmenting Techniques Classifications according to how they affect and transform the from 


\section{- References:}

1. Altuhafi "Application of Fractal Geometry in Islamic Architecture: An Analytical Study of Ottoman Mosques" - Journal of Civil Engineering and Architecture Research, Vol. 2, No. 1 - 2015

2. Benjamin Aranda, Chris Lanch "Pamphlet Architecture 27: Tooling" Princeton Architectural press, New York - 2006.

3. Bruna Ferreira "Construction \& Deconstruction in Architecture" - Published research - Issue.com - 2014.

4. Carl Bovill "Fractal Geometry in Architecture and Design" - Birkhauser Boston publisher - USA - 2015.

5. Donald P. Brown "Triangulation for accelerated rendering of polygons" United States Patent - USA - 2012.

6. Kenneth Falconer "Fractals a very short introduction" - Oxford university press - United Kingdom - 2013.

7. Kevin Li "Deconstruction: A True Form of Architecture" - Published research - Issue.com - 2015.

8. Philip Johnson \& Mark Wigley "Deconstructivist architecture" - Little Brown and Co - New York - 2005.

9. Robert Stuart "Erosion: Behavioral Matter Studio" - school of architecture United Kingdom, London - 2012.

10. Walter" W. Piegorsch "Fractal dimensions" - John Wiley \& Sons, Ltd - New York, USA - 2002.

11. Xiaoshu Lu "Fractal Geometry and Architecture Design: Case Study Review" - Published paper - Chaotic Modeling and Simulation (CMSIM) Journal Greece -2012 .

12. https://milford.nserl.purdue.edu/weppdocs/overview/wndersn.html

13. https://opentextbc.ca/geology/chapter/13-3-stream-erosion-and-deposition/

14. https://www.lifepersona.com/what-is-rain-erosion

15. https://architizer.com/blog/inspiration/collections/triangulated-facades-1/

16. https://www.slideserve.com/kenisha/triangulation-2d-3d

17. http://web.nateko.lu.se/courses/ngen06/Lectures/L8\%20Delaunay\%20triangu lation\%202016.pdf

18. https://robertstuart-smith.com/rs-sdesign-namoc-national-art-museum-ofchina

19. http://futuristicnews.com/crystal-like-lo-monaco-house-concept/ 